\title{
Short-term Forecast of Hourly Electricity Demand in Iran Using a Forecast Combination Method
}

\author{
Seyed Farshad Fatemi Ardestani ${ }^{1}$ | ffatemi@sharif.ir \\ Seyed Mahdi Barakchian² | m.barakchian@imps.ac.ir \\ Hamideh Shokoohian ${ }^{3}$
}

\begin{abstract}
The aim of this study is to present two time-series forecasting models and combine these models to provide a short-term prediction for hourly electricity demand, using daily electricity consumption data for the period 2006-2011. The first model is based on the decomposition of the electricity load into deterministic and stochastic components and the second model is based on the assumption that the electricity load is a stochastic time series. Once the hourly demand for electricity load is predicted using the above-mentioned models, the performance of the combined model is compared with the two time-series models and also with the dispatching unit model (a multi-variable model in which the weather variable is also included). The results show that the use of the combined model leads to an increase in prediction accuracy over the two time-series models. Moreover, the accuracy of the combined model is as good as the dispatching unit model for most of the time during the day, and even better during the consumption peak hours.
\end{abstract}

Keywords: Electricity Demand, Short-term Forecast, Forecast Combination, Time-series Modeling, Time-series Decomposition.

JEL Classification: L94, C53, C32.

\footnotetext{
1. Assistant Professor, Faculty of Management and Economics, Sharif University of Technology, Tehran, Iran. (Author Corresponding)

2. Assistant Professor, Institute for Management and Planning Studies, Tehran, Iran.

3. M.A. in Economics, Faculty of Management and Economics, Sharif University of Technology, Tehran, Iran.
} 


\section{ييشبينى كوتاهمدت تقاضاى برق به تفكيك

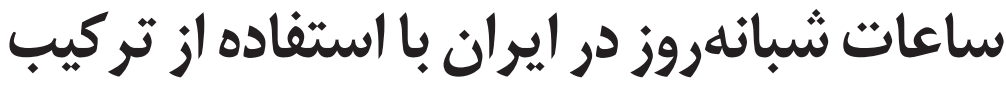

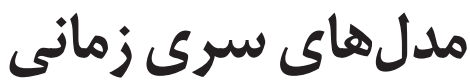

ffatemi@sharif.ir

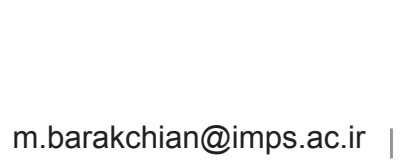

مقاله بزوهشى
سيدفرشاد فاطمى اردستانى

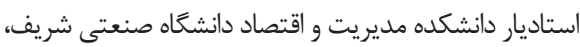
تهران، ايران.(نويسنده مسئول)

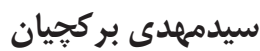

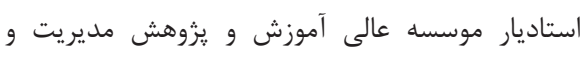

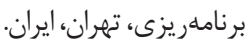

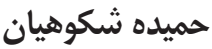
كارشناس ارشد علوم اقتصادى دانشكده اقتصاد و مديريت دانشعاه صنعتى شريف، تهران، ايران.

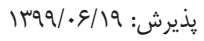

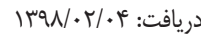

קكيده: هدف از يزوهش حاضر ارائه دو مدل بيشبينى سرى زمانى و همجنين، تركيب دو مدل

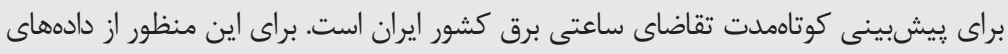

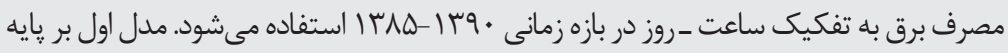

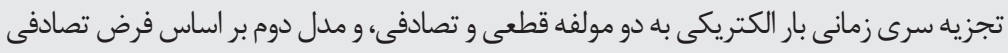

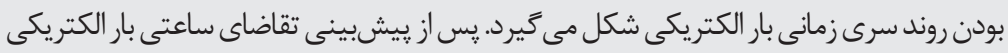

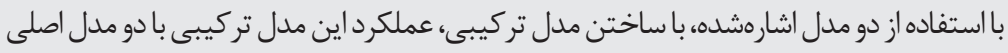

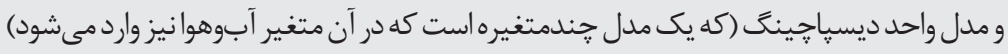

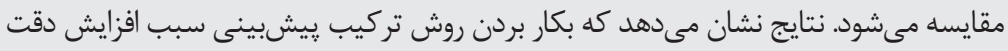

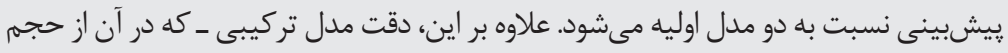

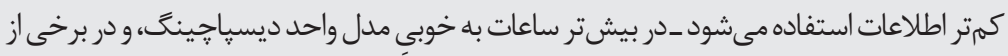

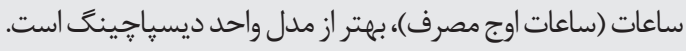

كليدوازهها: تقاضاى برق، پيشبينى كوتاهمدت، تركيب پِيشبينى، مدلسازى سرى زمانى، تجزيه سرى زمانى. طبقهبندى زمانى، تجزيه سرى زمانى. 


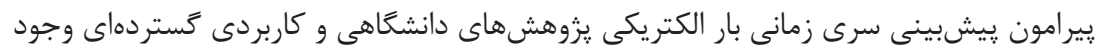

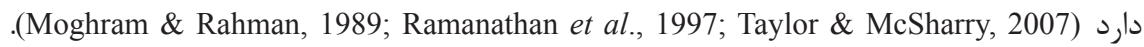
وجود روند، الكوهاى روزانه، فصلى، و سالانه اثر گذارى متغيرهاى بيرونى، و همجنين امكان غيرخطى بودن

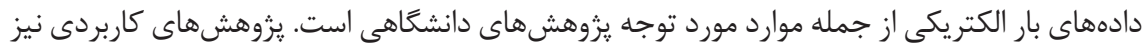
بيشتر به صرفهجويى هاى ناشى از دقت ييشبينى مصرف برق براى شركتهاى توليد كننده مى يردازند.

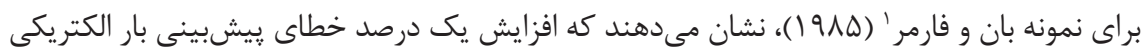

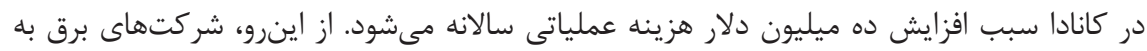

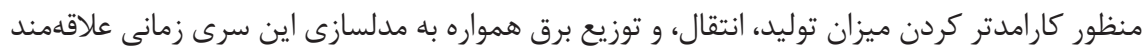

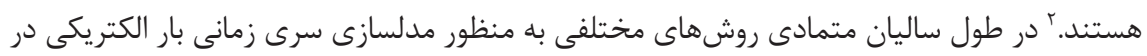

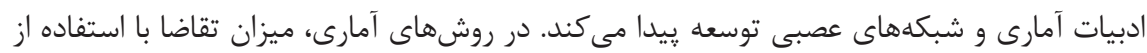

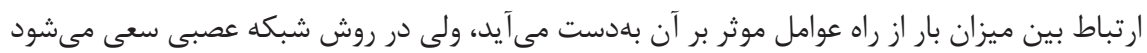

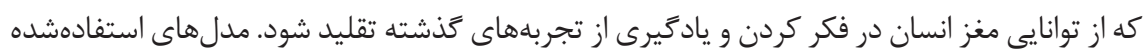

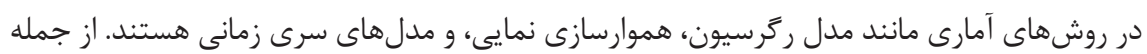

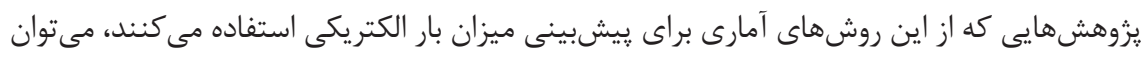

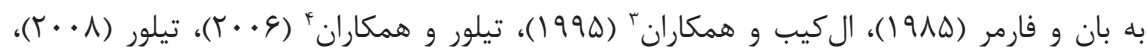

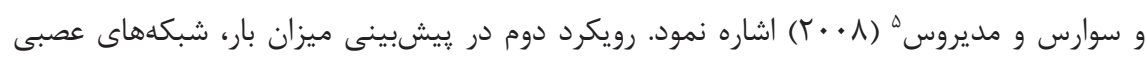
است. سيستمهاى خبره و سيستم منطق فازى از جمله مدلهايى هستند كه در اين رويكرد جاى

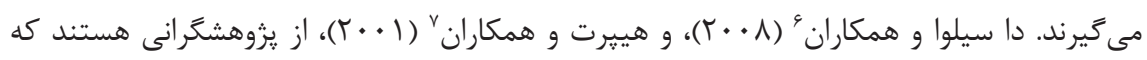

1. Bunn \& Farmer ז. با تصويب آييننامهاى توسط هيئت تنظيم بازار برق در سال M M ا اينَّنه هزينها در بازار برق ايران نيز

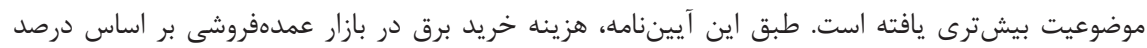

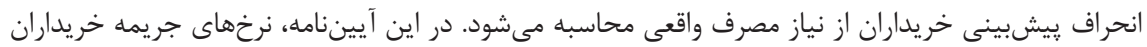

3 .El-Keib et al. در مقابل درصدهاى مختلف خطاى يِيشبينى بلهور كامل آمده است.

4. Taylor et al.

5. Soares \& Medeiros

6. Da Silva et al.

7. Hippert et al. 
از اين رويكرد استفاده مى كنند (همجنين براى مطالعه بيشبينى ميزان بار الكتريكى نكاه كنيد به Feinberg \& Genethliou, 2005 Alfares \& Nazeeruddin, 2002 براى پيشىبينى تقاضاى ساعتى بار الكتريكى مبتنى بر ادبيات سرى زمانى است. در اين يزوهش، ميزان

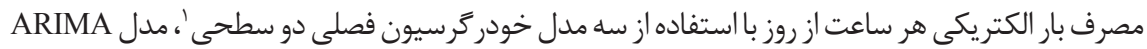

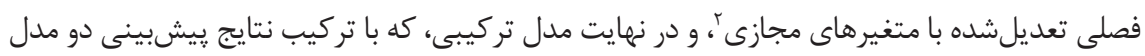

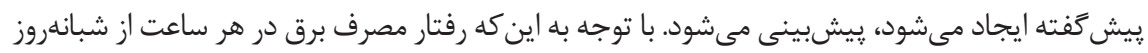

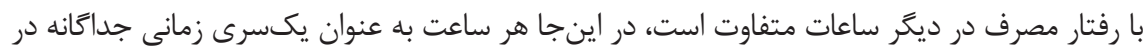

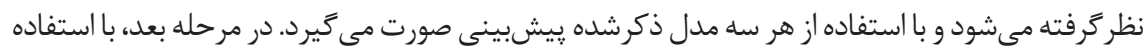

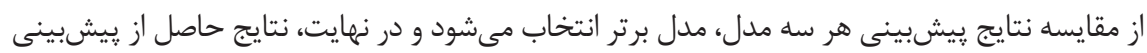

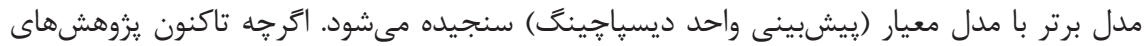

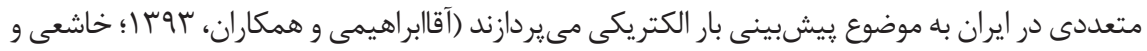

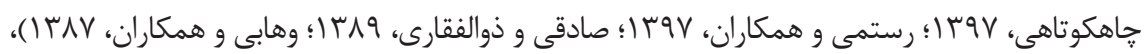
اما يروهش حاضر نخستين يزوهشى است كه به پيشبينى بار الكتريكى به تفكيك ساعات شبانهروز مى يردازد و عملكرد تركيب مدل هاى سرى زمانى را در يِيشبينى بار الكتريكى ايران ارزيابى ميى كند. نتايج

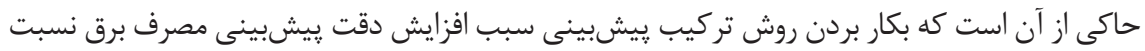

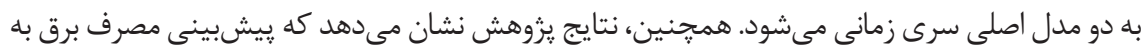

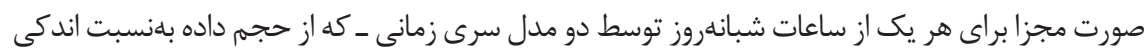

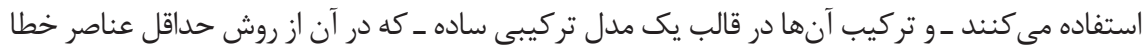

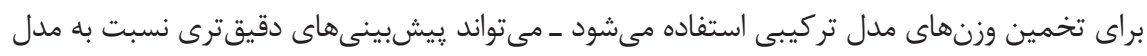

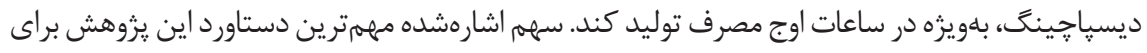

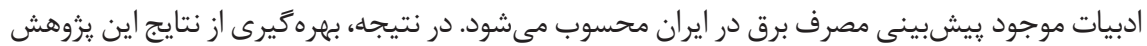
مى تواند در عمل به كاهش هزينههاى توليد برق منجر شود. در بخش بعد به معرفى دادههاى مورد استفاده و توضيح مدلهاى ييشبينى يرداخته مى شود.

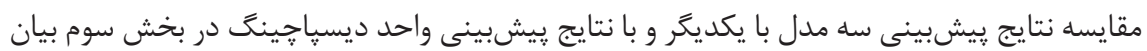
مىشود. بخش آخر نيز به بحث و نتيجه كيرى مى يردازد. 


\section{معرفى دادهها و مدلهاى ييشبينى}

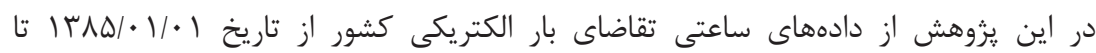

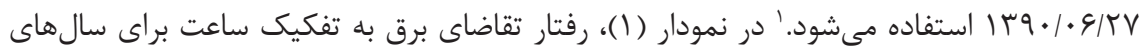

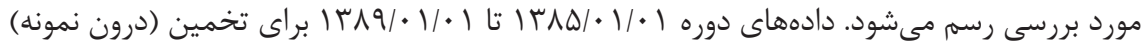
مدل خودرگرسيون فصلى دو سطحى (TLSAR)، و مدل ARIMA فصلى تعديلشده با متغيرهاى

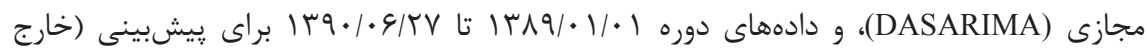
نمونه) استفاده مىشوند. به منظور ساختن مدل تركيبى نيز از نتايج يِيشبينى دو مدل در بازه

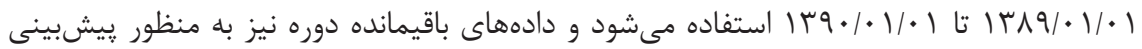
(خارج نمونه) بكار مىروند. - (خآن
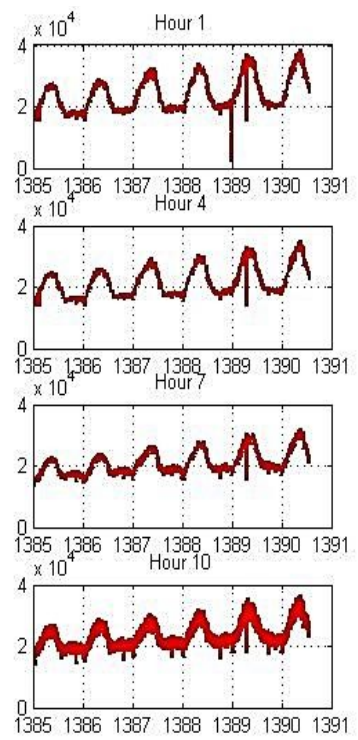
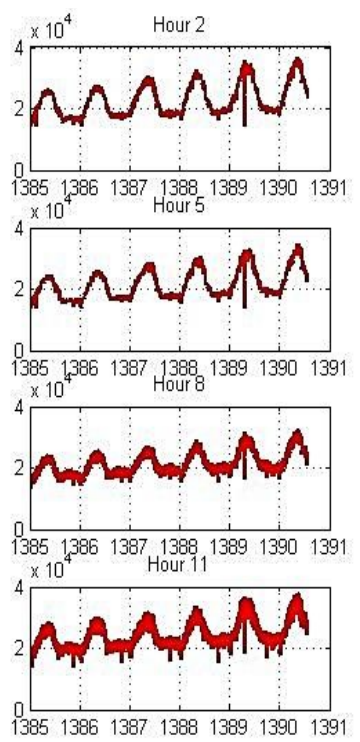
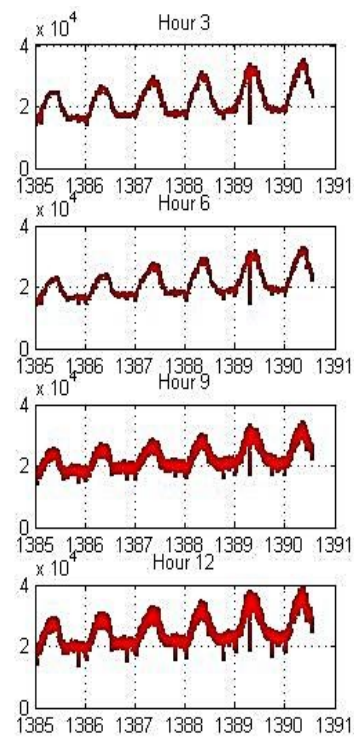

1. دادههاى مربوط به تقاضاى برق و همجنين پِيشبينىهاى واحد ديسياجينَ روى سايت شركت مديريت

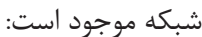
https://www.igmc.ir/electronic-services/power-market/reports/grid-load-forecast-report 

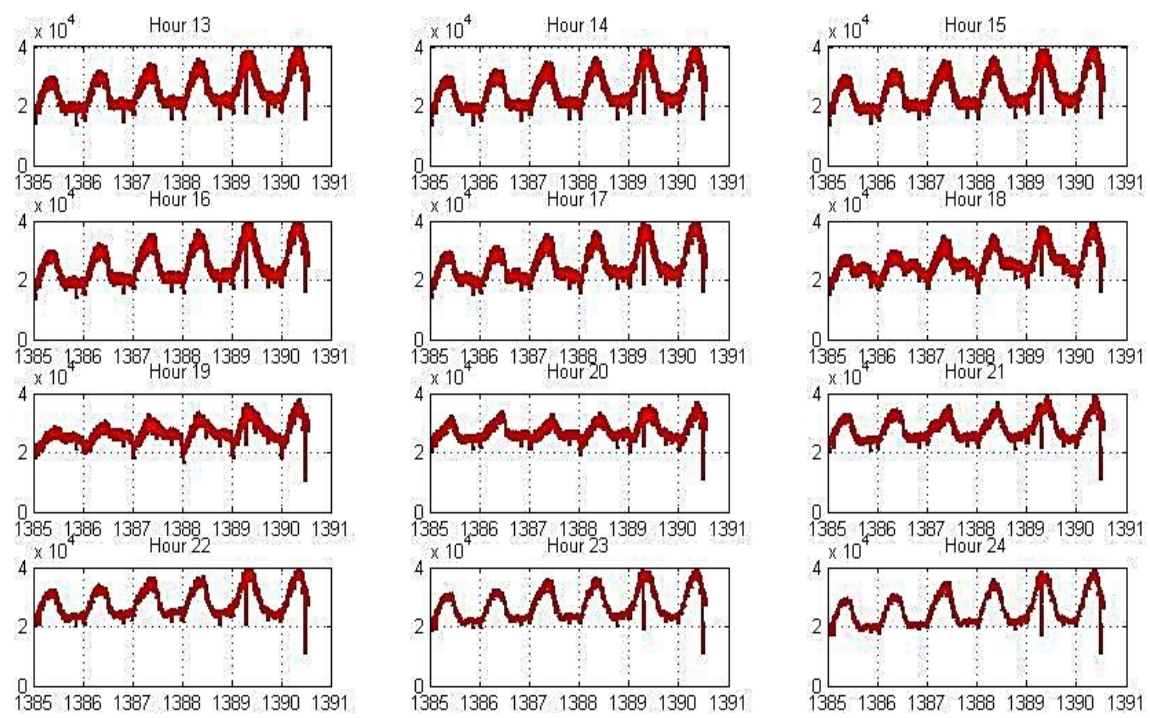

\begin{tabular}{ll}
1385 \\
$\times 10^{4}$ & 1386 \\
Hour 24 & 138913901391 \\
\hline
\end{tabular}

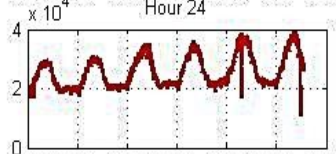

نمودار ا: دادههاى مصرف روزانه برق به تفكيك ساعت • وسا -هرسا (واحد مEاوات ساعت)

مدل TLSAR

رويكرد بكاررفته در مدل TLSAR مبتنى بر تجزيأ دومرحلهاى سرى زمانى بار الكتريكى است.

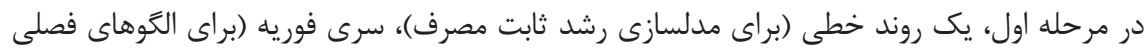

سالانه)، و متغيرهاى مجازى (براى اثر روزهاى مختلف هفته و مناسبتهاى تقويمى) تخمين زده مىشود.

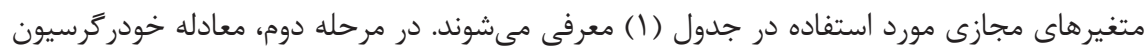

مناسب براى مدلسازى بخش تصادفى سرى زمانى تخمين زده مى معود (Temraz et al., 1996).

جدول ا: متغيرهاى مجازى بكاررفته در مدل TLSAR

\begin{tabular}{|c|c|}
\hline توصيف & متغير مجازى \\
\hline روزهاى هفته & $\delta_{1} \delta_{2} \ldots \delta_{7}$ \\
\hline تعطيلات رسمى و مذهبى & $\delta_{8}$ \\
\hline rا روز اول فروردين & $\delta_{9}$ \\
\hline تعطيلات بيشتر از دو روز & $\delta_{10}$ \\
\hline تعطيلات خاص & $\delta_{11}$ \\
\hline
\end{tabular}




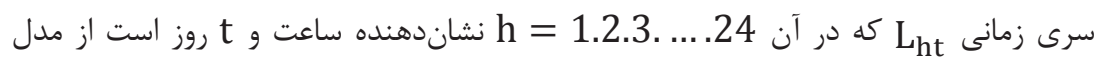
TLSAR

$\mathrm{L}_{\mathrm{ht}}=\mathrm{L}_{\mathrm{ht}}^{\mathrm{P}}+\mathrm{L}_{\mathrm{ht}}^{\mathrm{I}}$

$\mathrm{L}_{\mathrm{ht}}^{\mathrm{P}}=\rho \mathrm{t}+\sum_{\mathrm{r}=1}^{\mathrm{H}}\left[\alpha_{\mathrm{r}} \cos (\omega \mathrm{rt})+\beta_{\mathrm{r}} \sin (\omega \mathrm{rt})\right]+\sum_{\mathrm{i}=1}^{\mathrm{K}} \mu_{\mathrm{i}} \delta_{\mathrm{i}}$

$\mathrm{L}_{\mathrm{ht}}^{\mathrm{I}}=\Phi^{\prime} \mathrm{L}_{\mathrm{ht}-\mathrm{i}}+\varepsilon_{\mathrm{ht}}$

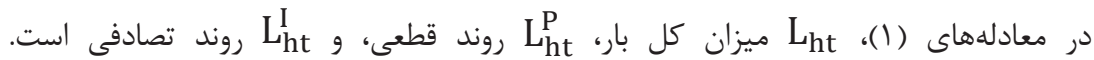

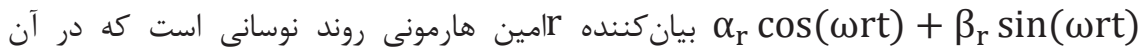

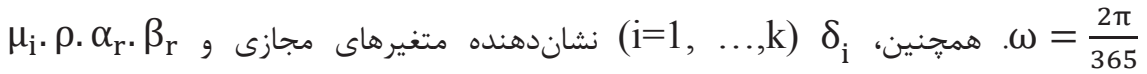

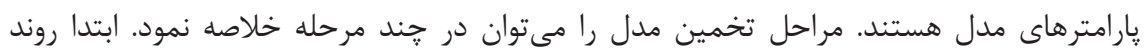

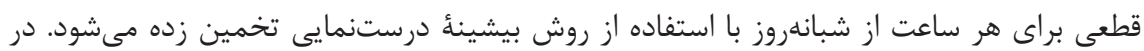

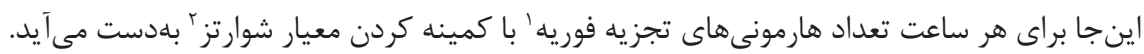

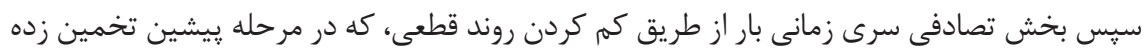
$\hat{\mathrm{L}}_{\mathrm{ht}}=\mathrm{L}_{\mathrm{ht}}-\hat{\mathrm{L}}_{\mathrm{ht}}^{\mathrm{p}}$

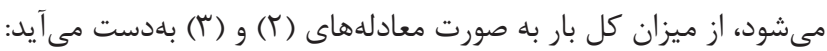

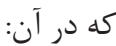

$\widehat{\mathrm{L}}_{\mathrm{ht}}^{\mathrm{p}}=\hat{\rho} \mathrm{t}+\sum_{\mathrm{r}=1}^{\mathrm{H}}\left[\widehat{\alpha}_{\mathrm{r}} \cos (\omega \mathrm{rt})+\widehat{\beta}_{\mathrm{r}} \sin (\omega \mathrm{rt})\right]+\sum_{\mathrm{i}=1}^{\mathrm{K}} \hat{\mu}_{\mathrm{i}} \delta_{\mathrm{i}}$

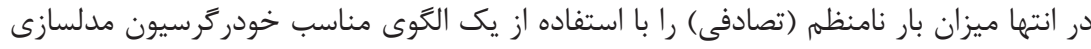

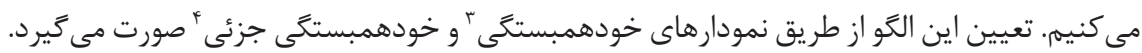

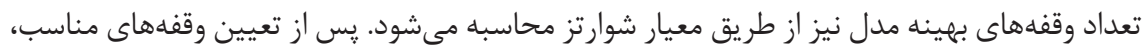
يارامترهاى مدل با استفاده از روش بيشينهٔ درستنمايى تخمين زده مىشود.

1. Fourier Decomposition

2. Schwartz

3. Autocorrelation

4. Partial Autocorrelation 


\section{مدل DASARIMA}

در اين يزوهش از مدل DASARIMA براى در نظرگرفتن روزهاى تعطيل و خاص استفاده

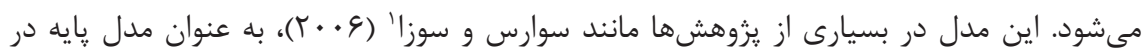
نظركرفته مىشود. مهممترين تفاوت اين است كه در مدل DASARIMA برخلاف مدل TLSAR فرض

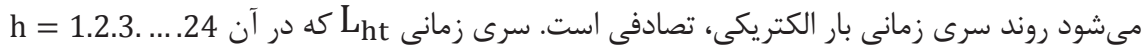

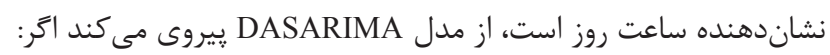
$\left(1-\phi_{1} B-\phi_{2} B^{2}-\cdots-\phi_{p} B^{p}\right) \Delta_{7} \Delta_{1} \bar{L}_{h t}=\left(1+\theta_{1} B+\cdots+\theta_{q} B^{q}\right)\left(1+\beta B^{7}\right) \varepsilon_{\text {h.t }}\left({ }^{q}\right)$ كه در آن: n (1) - n $\Delta_{7} \Delta_{1} \overline{\mathrm{L}}_{\mathrm{ht}}=\Delta_{7} \Delta_{1} \mathrm{~L}_{\mathrm{ht}}-\sum_{\mathrm{i}=1}^{\mathrm{M}} \alpha_{\mathrm{i}} \delta_{\mathrm{it}}-\sum_{\mathrm{i}=1}^{\mathrm{M}} \lambda_{\mathrm{i}} \delta_{\mathrm{it}-1}-\sum_{\mathrm{i}=1}^{\mathrm{M}} \gamma_{\mathrm{i}} \delta_{\mathrm{it}-7}$

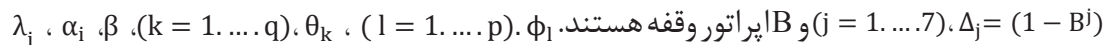

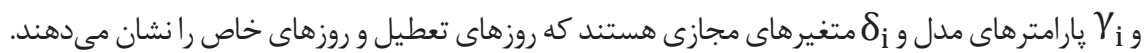

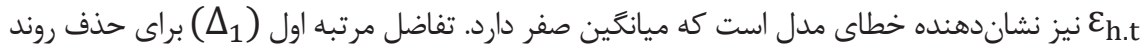

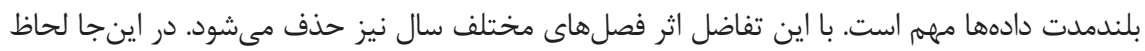

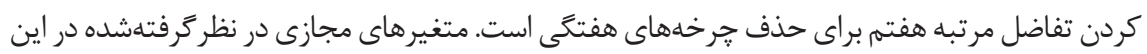

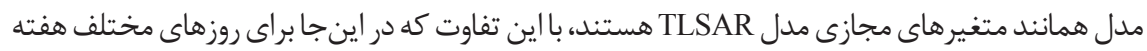

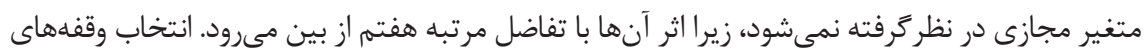
بهينه مانند مدل قبل بر اساس تحليل همبستخى و همبستخى جزئى و معيار شوارتز انجام مى مئيرد.

\section{تر كيب بيشبينى}

مفهوم تركيب بيشبينى، كه هدف از آن بهبود دقت يیشبينى است، به معناى تشكيل سبدى از ״يشبينى هاى مختلف است كه وزن بهينه هر يیشبينى در اين سبد با توجه به ماتريس واريانس ـ كواريانس

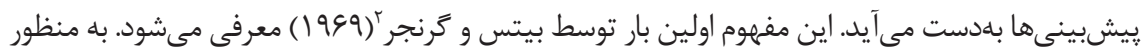

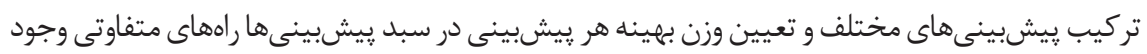

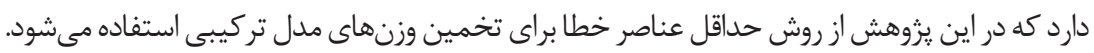




\section{نتايج تجربى}

روش يِشبينى بكاررفته در اين يزوهش يِيشبينى بروننمونهاى است. به اين معنا كه هر روز

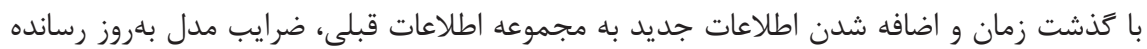
مىشوند. براى بررسى دقت ييشبينىهاى انجامگرفته توسط مدلهاى مختلف از معيار RMSFE' $\mathrm{L}\left(\mathrm{e}_{\mathrm{t}+\mathrm{h} \mid \mathrm{t}}^{\mathrm{i}}\right)=\left(\frac{1}{\mathrm{n}} \sum_{\mathrm{t}=1}^{\mathrm{n}}\left(\mathrm{e}_{\mathrm{t}+\mathrm{h} \mid \mathrm{t}}^{\mathrm{i}}\right)^{2}\right)^{1 / 2}$ استفاده مى شود: در جداول زير معيار RMSFE براى يِيشبينى مصرف برق در ساعتهاى مختلف و براى افقهاى

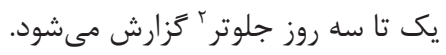

جدول r: RMSFE بيشبينى براى افق يك روز جلوتر

\begin{tabular}{|c|c|c|c|c|c|c|c|c|c|c|c|c|}
\hline دوازده & يازده & ده & نه & هشت & هفت & ششץ & ينج & جهار & سه & دو & يك & \\
\hline$r / l f$ & 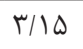 & $r / 9 V$ & r/99 & $r / T$ & I/V & $1 / 09$ & $1 / 94$ & $\mid / 91$ & $1 / 91$ & $1 / 99$ & $1 / 99$ & DASARIMA \\
\hline$r / \cdot$. & $r / r$ & r/Aq & r/gr & $r / \cdot G$ & $1 / 94$ & $1 / 09$ & $1 / 99$ & $1 / 9$ & $1 / 9$. & $1 / 99$ & T/r & TLSAR \\
\hline$r / \cdot r$ & $r / \cdot F$ & T/AV & $r / \Delta V$ & $r / 1 \Lambda$ & I/VT & $1 / 199$ & $1 / 94$ & $1 / 9$. & $1 / \Lambda \Lambda$ & $1 / 9 \Lambda$ & T/Tr & تركيب \\
\hline$r / . q$ & $r / T^{2}$ & $1 / \wedge \Lambda$ & $1 / V \Delta$ & l/94 & l/94 & N/AT & I/VT & I/VF & I/VV & I/AG & I/VG & ديسيا ֶֶينَ \\
\hline
\end{tabular}

\begin{tabular}{|c|c|c|c|c|c|c|c|c|c|c|c|c|}
\hline و و جهار & و بيست & و بيست & و بيست & بيست & نوزده & هجده & هفده & شانزده & يانزده & جهارده & سيزده & \\
\hline $1 / 9 V$ & I/AV & $1 / 99$ & $1 / 99$ & t/T & T/Tr & $r / \Delta \cdot$ & $r / \Lambda r$ & $r / 99$ & $r / \cdot \Lambda$ & $r / 9 \Lambda$ & r/lq & DASARIMA \\
\hline $1 / 90$ & I/^9 & $1 / 94$ & $1 / 19$ & $r / I V$ & T/IV & $r / F r$ & $r / 9 V$ & r/VA & r/AN & T/AF & $r / 99$ & TLSAR \\
\hline $1 / 9 r$ & l/Ar & $1 / 91$ & $1 / 19$ & $r / 19$ & T/Tr & $T / K T$ & T/VT & T/AT & $r / 94$ & T/AG & $r / \cdot r$ & تركيب \\
\hline$r / T^{c}$. & $T / T \cdot$ & $r / \cdot G$ & $r / \cdot I$ & $r / \cdot r$ & $r / r)$ & r/TN & $T / T V$ & $r / 19$ & $r / r \Lambda$ & $r / T V$ & $5 / 19$ & ديسياجينَ \\
\hline
\end{tabular}

1. Root Mean Square Forecast Error

ז. در اين يروهش، عملكرد مدلها در ماههاى مختلف سال و روزهاى مختلف هفته نيز مورد بررسى قرار

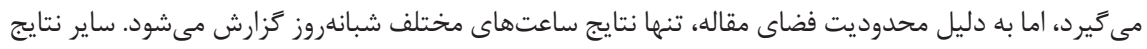
در نزد نويسندكان موجود است.

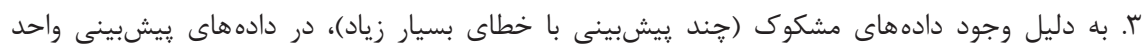

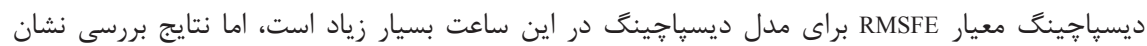

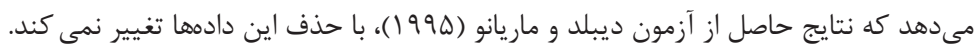




\section{جدول ": RMSFE ييشبينى براى افق دو روز جلوتر}

\begin{tabular}{|c|c|c|c|c|c|c|c|c|c|c|c|c|}
\hline دوازده & يازده & ده & نه & هشت & هفت & شش & ينج & جههار & سه & دو & يك & \\
\hline$r / F r$ & טגוr & $r / \cdot q$ & $r / \wedge \Delta$ & $r / F G$ & I/VT & T/Tr & $r / T \omega$ & r/Tr & t/Tr & $r / F r$ & $T / V I$ & DASARIMA \\
\hline T/l9 & r/T & $r / 9 \Delta$ & T/VT & T/V & $T / F \Delta$ & T/AT & r/gF & $r / \Delta K$ & $r / \Delta \mid$ & $r / 9$. & r/Ar & TLSAR \\
\hline$r / T)$ & $T / 10$ & $r / 94$ & $r / 9 \Lambda$ & T/KT & $r / I F$ & $r / 19$ & سז/r & T/TA & T/TA & $r / r q$ & $r / V \cdot$ & تركيب \\
\hline T/VG & $r / 9 q$ & $T / \Delta F$ & $r / T \Delta$ & $r / 1 \Delta$ & $1 / 99$ & N/94 & T/19 & $T / T \cdot$ & $r / T$. & $T / T I$ & $r / T V$ & ديسياجِ \\
\hline
\end{tabular}

\begin{tabular}{|c|c|c|c|c|c|c|c|c|c|c|c|c|}
\hline جيست و جهار & و بيست & و و دو & و بيست & بيست & نوزده & هجده & هفده & شانزده & يانزده & جهارده & سيزده & \\
\hline$r / V V$ & $r / 9 q$ & T/9V & r/GT & r/Ar & $r / / \Delta$ & $r / Y T$ & $r / \mu q$ & r/rq & r/fG & & r/FA & DASARIMA \\
\hline$r|9|$ & $r / 4 q$ & $r / T \Delta$ & 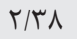 & $r / 9 \Lambda$ & r/.r & $r / T$ & $r / \cdot \Lambda$ & $r / \cdot \Delta$ & r/l. & $r / \cdot V$ & r/lV & TLSAR \\
\hline$r / V \Delta$ & $r / \Delta Y^{c}$ & $r|\Delta|$ & $r / F r$ & $r / V I$ & $r / \cdot r$ & $r / 19$ & $\mathrm{~T} / \mathrm{T}$ & 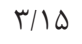 & r/l & س & $r / T F$ & تركيب \\
\hline$r / 9 V$ & r/gF & $r / 9 \wedge$ & $r|9|$ & T/99 & $r / 99$ & r/A & r/^९ & $r / \Lambda \cdot$ & $r / \wedge \Delta$ & $r / 9$. & T/AF & ديسياجينَ \\
\hline
\end{tabular}

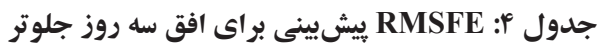

\begin{tabular}{|c|c|c|c|c|c|c|c|c|c|c|c|c|}
\hline دوازده & يازده & ده & نه & هشت & هفت & شش & رِنج & جهار & سه & دو & يك & ساعت \\
\hline r/Vq & $r / 99$ & T/QT & שr/r & $T / 9 T$ & T/9V & T/VD & $r / \cdot 1$ & $r / 9$. & T/AV & $r / \cdot 1$ & ז/זq & DASARIMA \\
\hline ז/ץq & $r / \mu r$ & r/Tr & $r / . q$ & $T / V V$ & $r / 90$ & $\mathrm{r} / 9 \mathrm{~T}$ & $r / I V$ & r/9V & र/9T & $r / \cdot \varphi$ & $\mathrm{r} / \mathrm{VV}$ & TLSAR \\
\hline$\mu / \mathcal{F} \Lambda$ & $r / \varphi$. & & $\mu / \cdot \Delta$ & $r / 9 \Lambda$ & $T / \Delta T$ & $r / 9 \Lambda$ & T/QT & r/Vq & T/VG & r/Aq & $r / \cdot q$ & تركيب \\
\hline$r / 90$ & $r / 4 \lambda$ & $r / F V$ & $r / r \Delta$ & T/9Y & $T / Q T$ & $9 / \cdot 9$ & $r / 99$ & r/Vq & $r / A \mid$ & $r / 9$. & r/9 & ديسياجِينَ \\
\hline
\end{tabular}

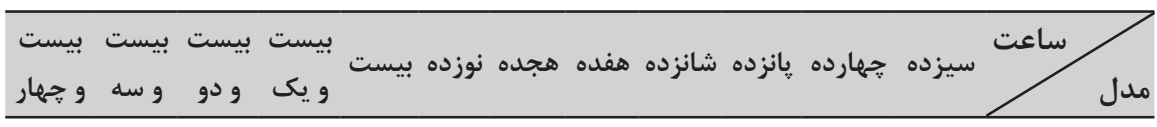

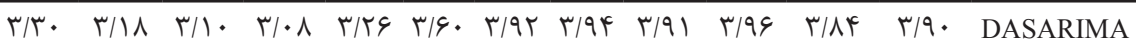

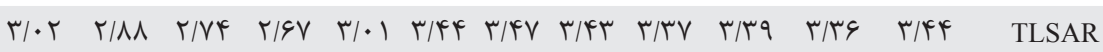

تركيب ديسياجينت تركيت 
با بررسى نتايج جداول بالا هند نكته قابلاستخراج است: الف. عملكرد يِشبينى هر سه مدل در ساعتهاى كمبارى (ساعت ا تا م)، بهتر از اوج بار

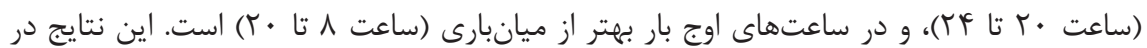
هر سه افق برقرار است و در نتيجه دقت ييشبينى هر سه مدل نسبت به افق يِيشبينى مقاوم است؛ ب. مدل TLSAR در ساعتهاى ميانبارى و اوج بار نسبت به مدل DASARIMA عملكرد بهترى

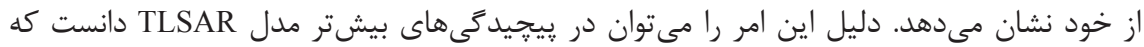
مناسب ساعتهاى ميانبارى و اوج بار است، زيرا در اين ساعتها الكَى مصرف نيز يِيجيدهتر است

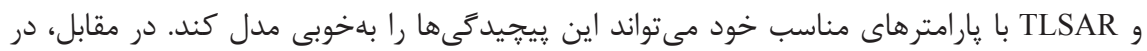

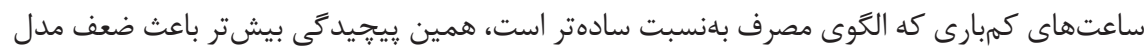

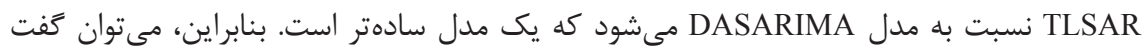

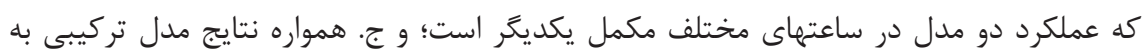

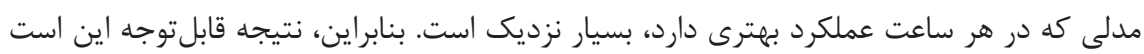

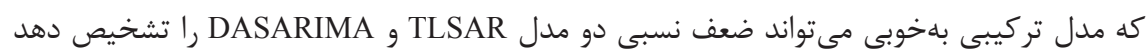
و در هر ساعت مدل بهتر را انتخاب كند. اين نتيجه نشان مىدهد، روش حداقل عناصر خطا ـ كه

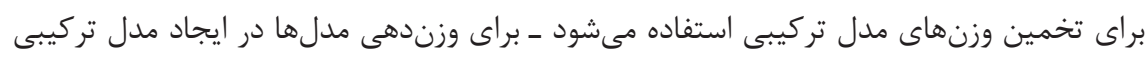

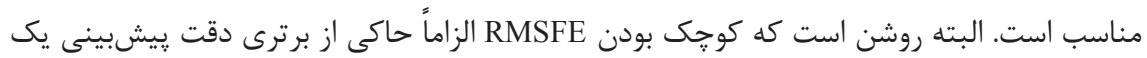

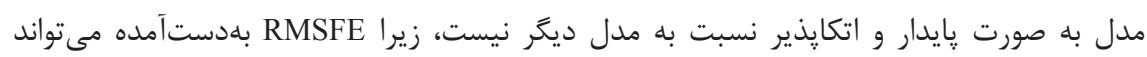

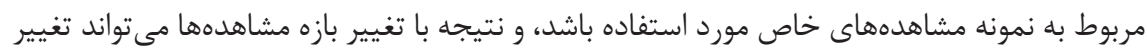

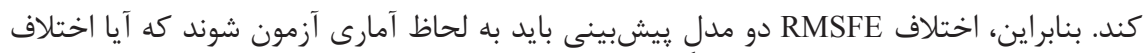

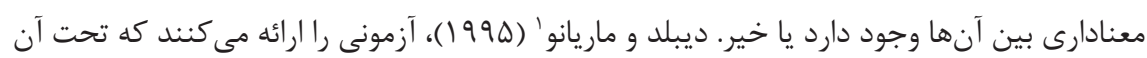

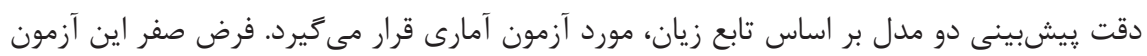
$\left\{\begin{array}{l}H_{0}: E\left[M\left(e_{t+h \mid t}^{1}\right)-M\left(e_{t+h \mid t}^{2}\right)\right]=0 \\ H_{1}: E\left[M\left(e_{t+h \mid t}^{1}\right)-M\left(e_{t+h \mid t}^{2}\right)\right] \neq 0\end{array}\right.$ به صورت تابع (V) نوشته مىشود:

$$
M_{t}\left(e_{t+h \mid t}^{i}\right)=\left(e_{t+h \mid t}^{i}\right)^{2} \text { (^) }
$$




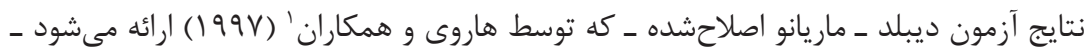

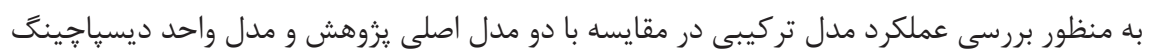
در يیيوست كزارش مىشود. نتايج مقايسه مدل تركيبى با دو مدل اصلى نشان مىدهد كه در برخى

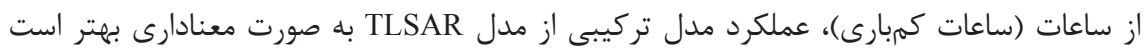
و به خوبي مدل DASARIMA است. از سوى ديكر، در ساعات اوج بار عملكرد اين مدل از مدل DASARIMA به صورت معنادارى بهتر است و با دقت مدل TLSAR برابرى مى كند. در نتيجه، مىتوان كَفت كه در مجموع مدل تركيبى نسبت به دو مدل اصلى سرى زمانى از دقت بالاترى

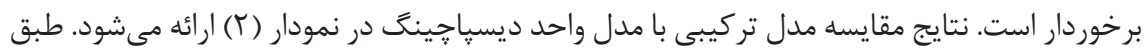

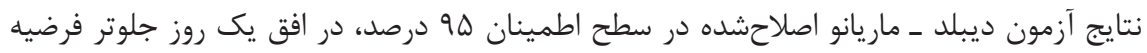

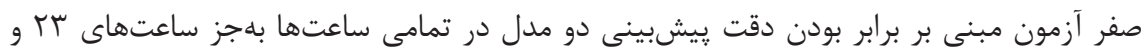

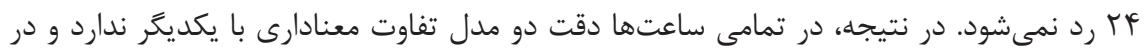

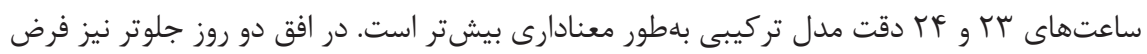

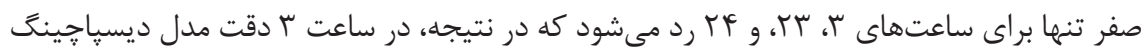
و در ساعتهاى س و و Y دقت مدل تركيبى به صورت معنادارى بيشتر است. در افق سه روز جلوتر هم فرض صفر در هيج يك از ساعتها رد نمىشود. 


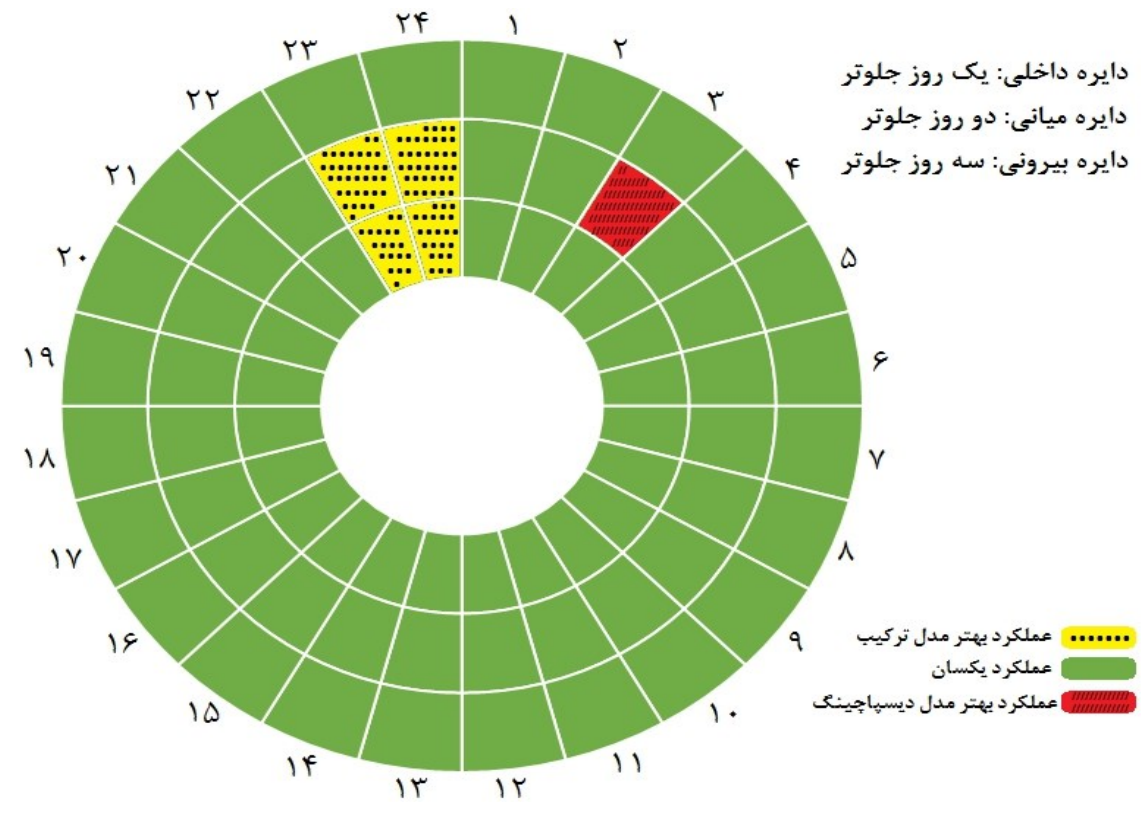

نمودار ז: مقايسه عملكرد مدل تر كيب با مدل واحد ديسياجِينَك بر اساس آزمون ديبلد ـ ماريانو

بحث و نتيجه

در يزوهش حاضر، تقاضاى ساعتى برق كشور با استفاده از دو مدل DASARMA و TLSAR و تركيب اين دو مدل پيشبينى مىشود، و دقت پيشبينى ها به وسيله معيار RMSFE مورد بررسى قرار

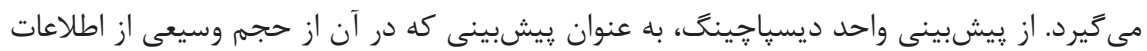

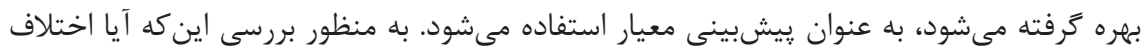
RMSFE

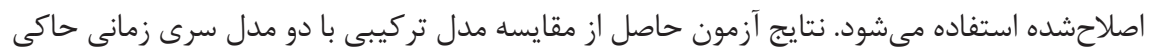

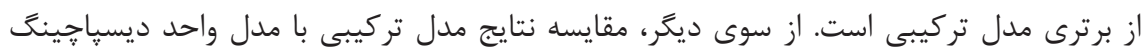

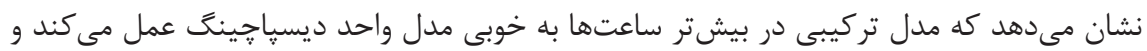

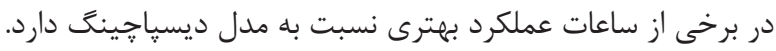




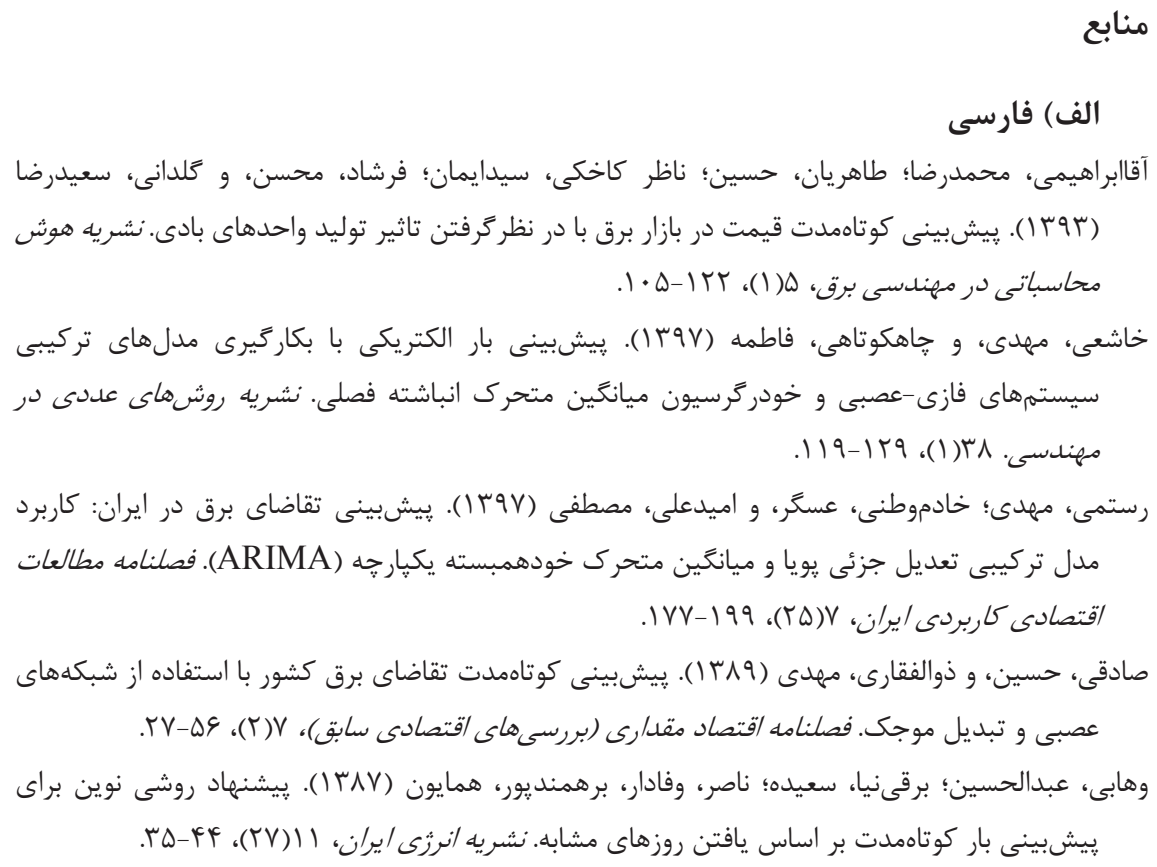

Alfares, H. K., \& Nazeeruddin, M. (2002). Electric Load Forecasting: Literature Survey and Classification of Methods. International Journal of Systems Science, 33(1), 23-34.

Bates, J., \& Granger, C. (1969). The Combination of Forecasts. Operations Research Quarterly, 20(1), 451-468.

Bunn, D., \& Farmer, E. (1985). Review of Short-Term Forecasting Methods in the Electric Power Industry. Comparative Models for Electrical Load Forecasting, 13-30.

Da Silva, A. P. A., Ferreira, V. H., \& Velasquez, R. M. (2008). Input Space to Neural Network Based Load Forecasters. International Journal of Forecasting, 24(4), 616-629.

Diebold, F., \& Mariano, R. (1995). Comparing Predictive Accuracy. Journal of Business and Economics Statistics, 13(1), 253-263.

El-Keib, A., Ma, X., \& Ma, H. (1995). Advancement of Statistical Based Modeling Techniques for Short-Term Load Forecasting. Electric Power Systems Research, 35(1), 51-58.

Feinberg, E. A., \& Genethliou, D. (2005). Load Forecasting. Applied Mathematics for Restructured Electric Power Systems (pp. 269-285): Springer.

Harvey, D., Leybourne, S., \& Newbold, P. (1997). Testing the Equality of Prediction Mean Squared Errors. International Journal of Forecasting, 13(2), 281-291. 
Hippert, H. S., Pedreira, C. E., \& Souza, R. C. (2001). Neural Networks for Short-Term Load Forecasting: A Review and Evaluation. IEEE Transactions on Power Systems, 16(1), 44-55.

Moghram, I., \& Rahman, S. (1989). Analysis and Evaluation of Five Short-Term Load Forecasting Techniques. IEEE Transactions on Power Systems, 4(4), 1484-1491.

Ramanathan, R., Engle, R., Granger, C. W., Vahid-Araghi, F., \& Brace, C. (1997). ShortRun Forecasts of Electricity Loads and Peaks. International Journal of Forecasting, 13(2), 161-174.

Soares, L. J., \& Medeiros, M. C. (2008). Modeling and Forecasting Short-Term Electricity Load: A Comparison of Methods with an Application to Brazilian Data. International Journal of Forecasting, 24(4), 630-644.

Soares, L. J., \& Souza, L. R. (2006). Forecasting Electricity Demand Using Generalized Long Memory. International Journal of Forecasting, 22(1), 17-28.

Taylor, J. W. (2008). An Evaluation of Methods for Very Short-Term Load Forecasting Using Minute-By-Minute British Data. International Journal of Forecasting, 24(4), 645-658.

Taylor, J. W., \& McSharry, P. E. (2007). Short-Term Load Forecasting Methods: An Evaluation Based on European Data. IEEE Transactions on Power Systems, 22(4), 2213-2219.

Taylor, J. W., De Menezes, L. M., \& McSharry, P. E. (2006). A Comparison of Univariate Methods for Forecasting Electricity Demand Up to a Day Ahead. International Journal of Forecasting, 22(1), 1-16.

Temraz, H., Salama, M., \& Quintana, V. (1996). Application of the Decomposition Technique for Forecasting the Load of a Large Electric Power Network. IEE ProceedingsGeneration, Transmission and Distribution, 143(1), 13-18. 
جدول اب: های آزمون ديبلد ـ ماريانو اصلاحشده براى مقايسه مدل تر كيبى با مدل

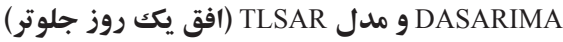

\begin{tabular}{|c|c|c|c|c|c|c|c|c|c|c|c|c|}
\hline دوازده & يازده & נه & نه & هشت & هفت & شش & ينج & جههار & سه & دو & يك & ساعت \\
\hline$\cdot 1 \cdot f$ & $\cdot 1 \cdot r$ & $\cdot 1 \cdot r$ & $\cdot 1 \cdot r$ & .119 & $\cdot \mid \Lambda \Lambda$ & $\cdot 10$. & .199 & $\cdot \mid \Delta T$ & $\cdot / T F$ & $\cdot|\Delta|$ & $\cdot 1 \cdot 1$ & وقايسه مدل تركيبى مدل DASARIMA \\
\hline$\cdot / \wedge \Delta$ & $\cdot / \vee \wedge$ & $\cdot 190$ & $\cdot / \mu r$ & $\cdot|r|$ & $\cdot 1 \cdot V$ & $\cdot 1 \cdot f$ & $\cdot 1 \cdot r$ & $\cdot 1 \cdot r$ & $\cdot 1 \cdot r$ & $\cdot 1 \cdot r$ & .191 & مقايسه مدل تركيبى مدل TLSAR \\
\hline
\end{tabular}

\begin{tabular}{|c|c|c|c|c|c|c|c|c|c|c|c|c|}
\hline و وجهار & بيست & بيست & و بيست & بيست & نوزده & هجده & هفده & شانزده & يانزده , & جههارده & سيزده & ساعت \\
\hline$\cdot|V|$ & $\cdot / \Delta \Delta$ & $\cdot / 1 F$ & $\cdot 1 \cdot r$ & $\cdot 1 \cdot r$ & $\cdot / \cdot \cdot$ & $\cdot 1 \cdot 1$ & $\cdot 1 \cdot r$ & $\cdot 1 \cdot r$ & $\cdot 1 \cdot r$ & $\cdot 1 \cdot r$ & $\cdot 1 \cdot r$ & تركيبى و مدل مدل مدل \\
\hline$\cdot / T \Lambda$ & $\cdot / T V$ & • & $\cdot|\Delta|$ & $\cdot / V T$ & • & $.19 V$ &.$|9|$ & $\cdot / \Lambda$. & $.19 \mathrm{~V}$ & $\cdot \mid \Lambda F$ & • & مقايسه مدل تركيبى مدل TLSAR \\
\hline
\end{tabular}

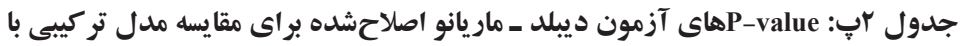

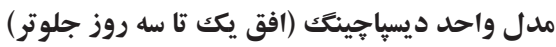

\begin{tabular}{|c|c|c|c|c|c|c|c|c|c|c|c|c|}
\hline دوازده & يازده & ده & نه & هشت & هفت & شش & ينج & جهار & سه & دو & يك & \\
\hline$\cdot 110$ & $\cdot 119$ & .119 & $\cdot / 1 \Delta$ & . &. $\mid 94$ & $\cdot / T$. & $\cdot \mid \Delta \varphi$ & $\cdot / \pi \Delta$ & $\cdot / T T$ &.$/ 1 \mathrm{~F}$ & $\cdot / 1 \mathrm{~V}$ & يك روز جلوتر \\
\hline •/rq & $\cdot \pi \Delta$ & $\cdot|f|$ & $\cdot \pi \Delta$ & . & $\cdot 119$ & $\cdot / T \cdot$ & $\cdot / r q$ & $\cdot 1 \Delta \Delta$ & $\cdot 109$ & $\cdot \mid \Delta F$ & $\cdot / \cdot r$ & دو روز جلوتر \\
\hline$\cdot 199$ & $\cdot / \wedge \Delta$ & . & $\cdot|9|$ & $\cdot / r \Lambda$ & .199 & $\cdot / \pi \cdot$ & $\cdot \pi r$ & .199 & $\cdot|V|$ & .198 & $\cdot / 4 \Delta$ & سه روز جلوتر \\
\hline
\end{tabular}

\begin{tabular}{|c|c|c|c|c|c|c|c|c|c|c|c|c|}
\hline و جيستار & و و سه & و دو & و بيست & يست & نوزده & & هفده & & & & & \\
\hline$\cdot 1 \cdot$ & $\cdot / \cdot \cdot$ & $\cdot / r$. & $\cdot / r V$ & $\cdot \mid \Delta T$ & . IFT & . /Tr & $\cdot|\pi|$ &.$/ 1 Y$ & .119 & . &.$/ 1 \mathrm{~V}$ & يك روز جلوتر \\
\hline$\cdot 1 \cdot 4$ & r./. & .1 .9 & $\cdot / T F$ & $\cdot / V \wedge$ & - |AT & $\cdot|r|$ & • $/ \pi V$ & $\cdot \pi \cdot$ & זr/. & $\cdot 10$. & $\cdot / \pi \Delta$ & دو روز جلوتر \\
\hline$\cdot 1 \cdot \Delta$ & .111 & $\cdot / T T$ & D MT & $\cdot \mid \wedge \Delta$ & $\cdot / V V$ & .191 & $\cdot / 90$ & .190 & .190 & $\cdot / \Lambda$. & - / 99 & سه روز جلوتر \\
\hline
\end{tabular}



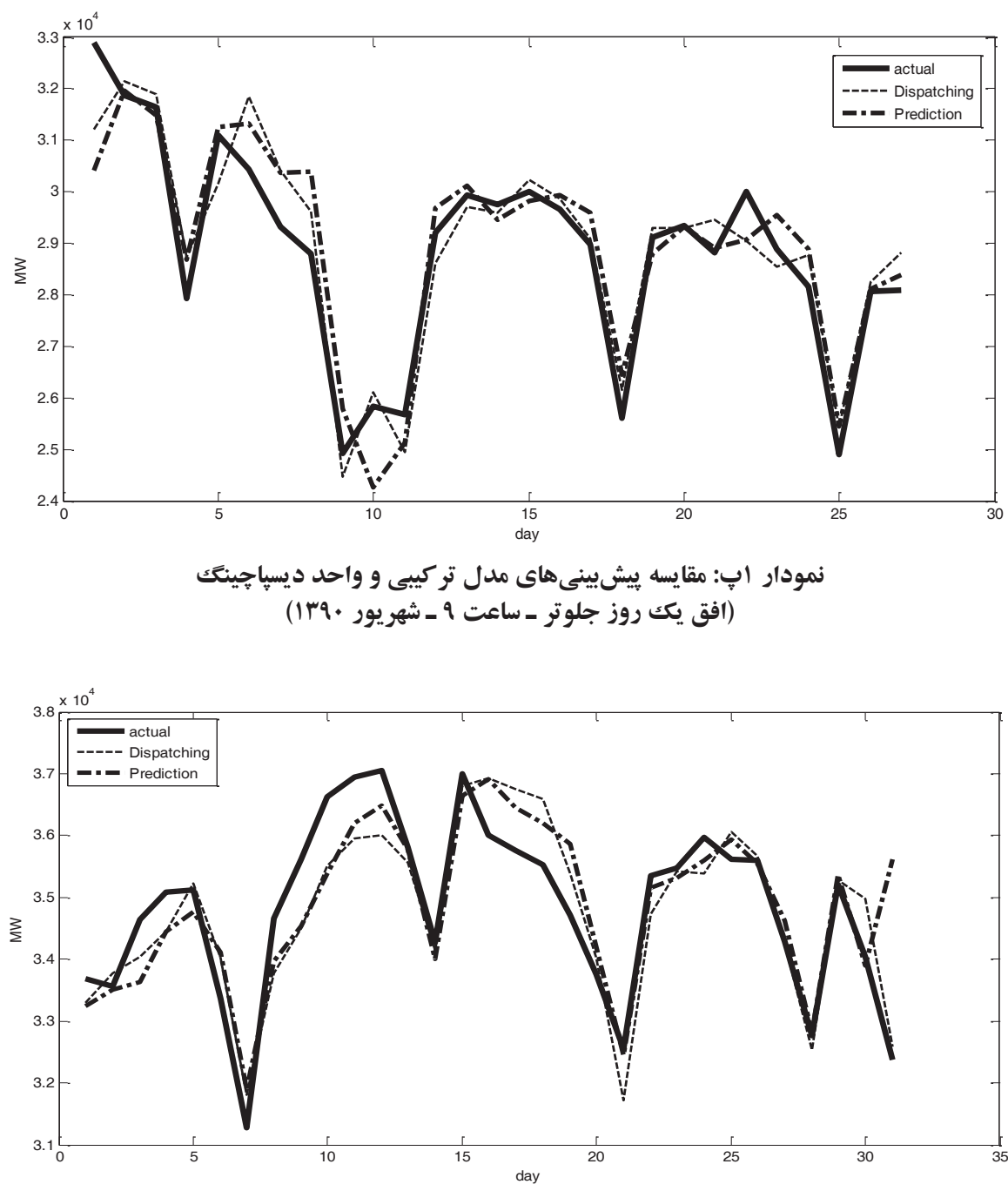

3
3
0
2
3
3
3
3
3
3
3

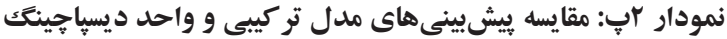

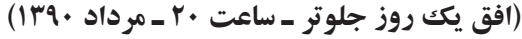




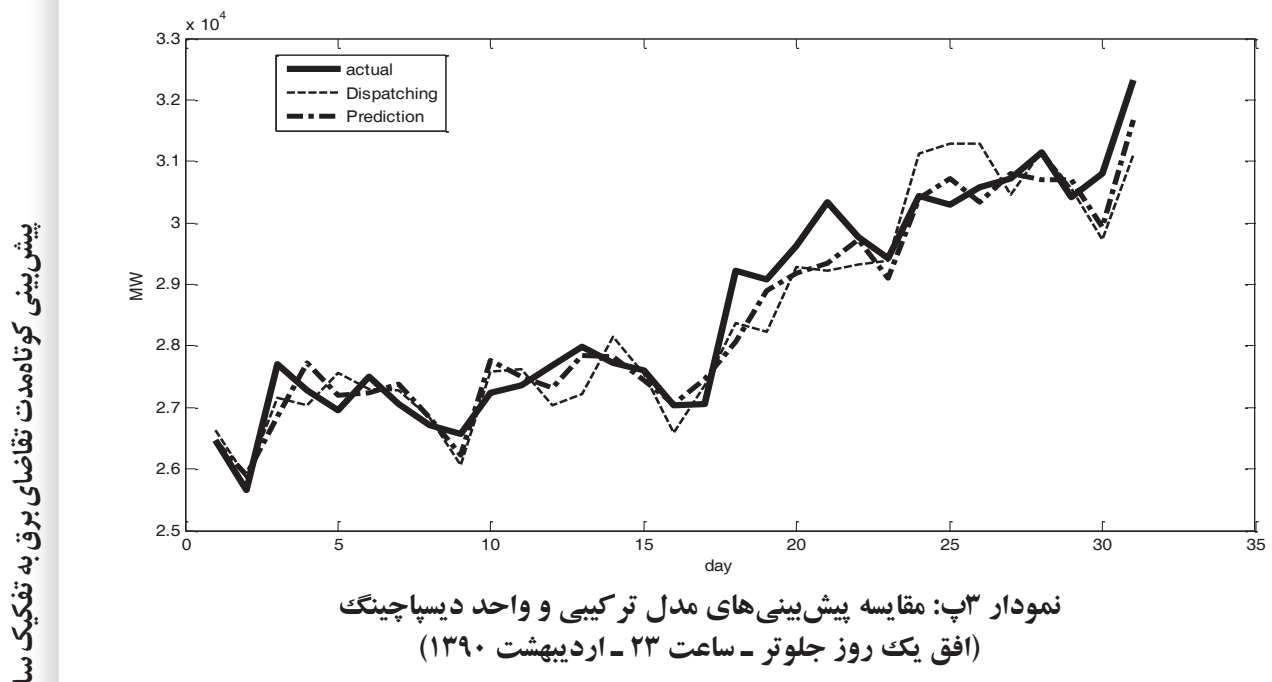

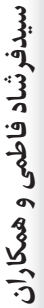

